\title{
Recent highlights from spin structure study of proton in PHENIX experiment at RHIC
}

\author{
Itaru Nakagawa for the PHENIX Collaboration* \\ RIKEN \\ 2-1 Hirosawa, Wako, Saitama 351-0198 \\ E-mail: itarueriken.jp
}

The Relativistic Heavy Ion Collider (RHIC) is a versatile machine that has provided collision between a wide range of heavy ions, from $\mathrm{d}$ to $\mathrm{Au}$, as well as proton+proton and proton+nucleus collisions. Polarized proton program at RHIC provides unique testing ground for the fundamental study of the proton's spin structure. The large transverse single spin asymmetry (TSSA) in pion production in high energy polarized proton+proton reaction was discovered almost 3 decades ago and the origin of the asymmetry is the one of the long standing open questions in QCD as of now. The latest highlights from the PHENIX experiment addressing the transverse spin structures of proton and some results from polarized proton at $\sqrt{s}=200 \mathrm{GeV}$ using various observables in wide rapidity region are discussed in this report. In addition, the latest TSSA results from transversely polarized proton+nucleus collision from Run15 are presented. Possible mechanism of rather unexpected large A-dependence in (very) forward rapidity region is addressed as well.

The European Physical Society Conference on High Energy Physics

5-12 July, 2017

Venice

${ }^{*}$ Speaker. 


\section{INTRODUCTION}

The transverse single spin asymmetry (TSSA) has been intensely studied in polarized proton+proton interaction in high energy field ever since unexpectedly large asymmetries are discovered in forward pion production at FNAL[四]. After decades of theoretical efforts, two formalisms have been proposed within perturbative QCD framework to explain the origin of large TSSAs at forward rapidity. Both formalisms connect the transverse motion of the parton inside the nucleon and/or to the spin-dependent quark fragmentation. The PHENIX collaboration explored various TSSA observables in wide rapidity regions from central to the very forward rapidity regions including the above mentioned "forward" rapidity region. The production mechanism is considered to be tightly connected with the rapidity of the observables. Especially the reactions are rather driven by the diffractive nature in the very forward rapidities unlike the central rapidity region. In addition to the rapidity dependence of TSSA, nuclear dependence of TSSA is also discussed as we executed the first polarized proton+nucleus collisions at high energy in Run15. In the following sections, selected highlights in each rapidity regions are introduced in the order of increasing rapidity.

\section{Central Rapidity}

The observables in central rapidity region at RHIC energy is in general considered to be sensitive to the reaction mechanism in partonic level. Due to the momentum balance between two colliding partons, gluons often play important role especially in low $p_{\mathrm{T}}$ region. No experimental evidence of finite $A_{\mathrm{N}}$ is found yet in high energy polarized hadron experiments so far. Shown in Fig. $\mathrm{W}$ is the observed analyzing power $A_{\mathrm{N}}$ of $\pi^{0}$ productions in $\mathrm{p}+\mathrm{p}$ (top), $\mathrm{p}+\mathrm{Al}$ (middle), and $\mathrm{p}+\mathrm{Au}$ (bottom) collisions at $\sqrt{s}=200 \mathrm{GeV}$ in central $(-0.35<\eta<0.35)$ rapidity region plotted as a function of $p_{\mathrm{T}}$. The asymmetry is expected to be sensitive to gluon Sivers twist-3 counter part though, the observed asymmetries are consistent with zero for all 3 collision systems within the statistical uncertainties.

\section{Forward Rapidity}

The PHENIX detector is implemented with dedicated muon detection system [四] in the forward rapidity region $(1.2<y<2.4)$. The transverse-momentum-dependent framework requires two momentum scales, and so is applicable to $\mathrm{W}$, DY , and $\mathrm{Z}$ production in $\mathrm{p}+\mathrm{p}$ collisions at RHIC. On the other hand, the collinear factorization framework needs only one scale $\left(Q^{2}\right.$ or $\left.p_{\mathrm{T}}\right)$, and is applicable to many observables, such as jet, heavy flavor in $\mathrm{p}+\mathrm{p}$ collisions at RHIC. Heavy flavor production is a great tool for investigating the gluon distribution in the proton; especially the effect of pure gluonic correlation functions on D meson production in transversely polarized $p+p$ is studied extensively as a twist-3 observable in the collinear factorization framework. Shown in Fig. $\square$ is the observed analyzing power $A_{\mathrm{N}}$ of $\mu^{-}$(left) and $\mu^{+}$(right) from open heavy-flavor decays mainly from D-mesons in $\mathrm{p}+\mathrm{p}$ collision at $\sqrt{s}=200 \mathrm{GeV}$ in forward rapidity region plotted as a function of $x_{\mathrm{F}}$ [B]. Because heavy-flavor production is dominated by gluon-gluon interactions, these offer a 

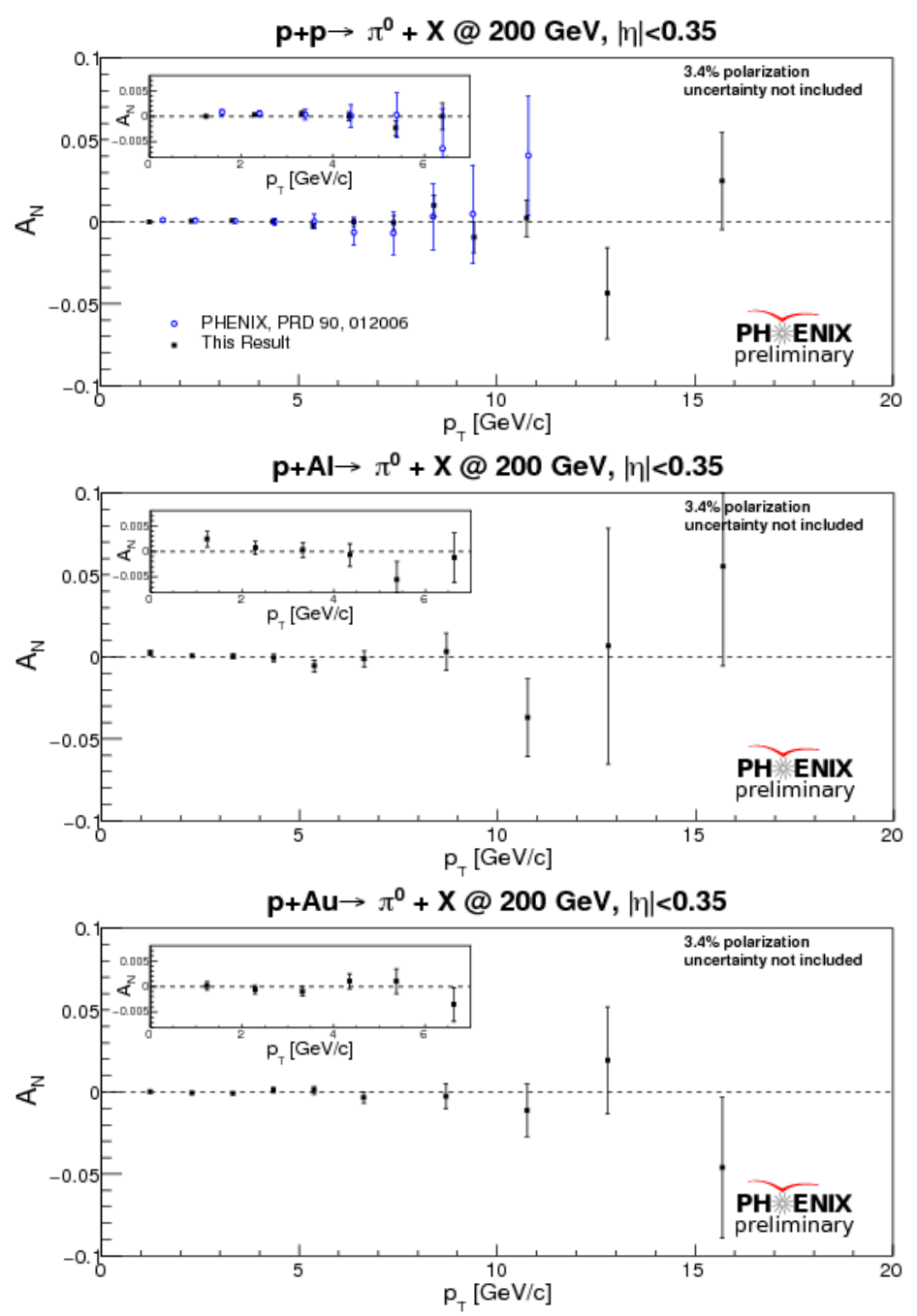

Figure 1: Observed analyzing power $A_{\mathrm{N}}$ of $\pi^{0}$ productions in $\mathrm{p}+\mathrm{p}$ (top), $\mathrm{p}+\mathrm{Al}$ (middle), and $\mathrm{p}+\mathrm{Au}$ (bottom) collisions at $\sqrt{s}=200 \mathrm{GeV}$ in central $(-0.35<\eta<0.35)$ rapidity region plotted as a function of $p_{\mathrm{T}}$.

unique test on the tri-gluon correlation functions. Although theory curves which calculates twist3 three gluon correlation function [5] by Koike and Yoshida predicts non-zero asymmetries and reproduce all experimental data, data are also consistent with zero within $2 \sigma$.

The $A_{\mathrm{N}}$ for $J / \psi$ production in forward/backward rapidity regions in polarized $\mathrm{p}+\mathrm{p}$ collision also shows almost zero consistent asymmetries as shown in black open circles in Fig. [1]. A data point around $p_{\mathrm{T}}$ at $3 \mathrm{GeV}$ in backward rapidity shows finite positive asymmetry within $2 \sigma$ though, further confirmation with more sufficient statistics is necessary to be conclusive. On the other hand, rather negative asymmetries about $A_{\mathrm{N}} \sim-0.1$ are observed for $p_{\mathrm{T}} \sim 1 \mathrm{GeV}$ data points in $\mathrm{p}+\mathrm{Au}$ collision as shown in solid red circles for both backward and forward asymmetries.

It is not yet clear if it is the same origin with the finite $A_{\mathrm{N}}$ observed for rather $J / \psi$ in small $p_{\mathrm{T}}$ though, an interesting observation was reported by ALICE collaboration in the forward $J / \psi$ production at $\sqrt{s}=2.76 \mathrm{TeV}\left[\right.$ [6]. Shown in Fig. 团 is the nuclear modification factor $R_{\mathrm{AA}}$ in $\mathrm{Pb}+\mathrm{Pb}$ 


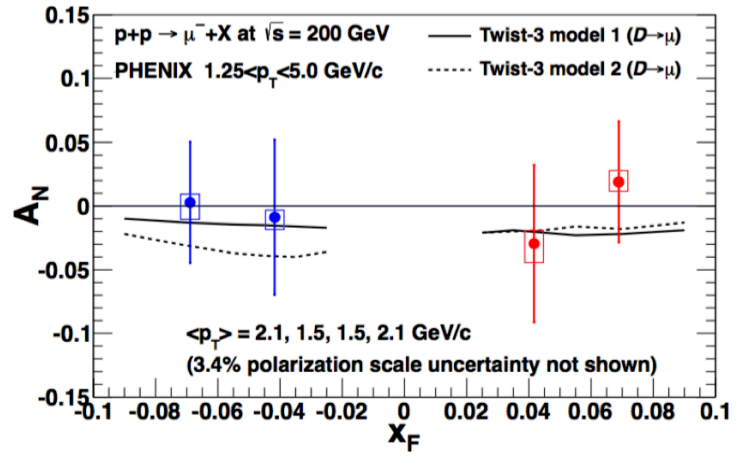

(-) charge

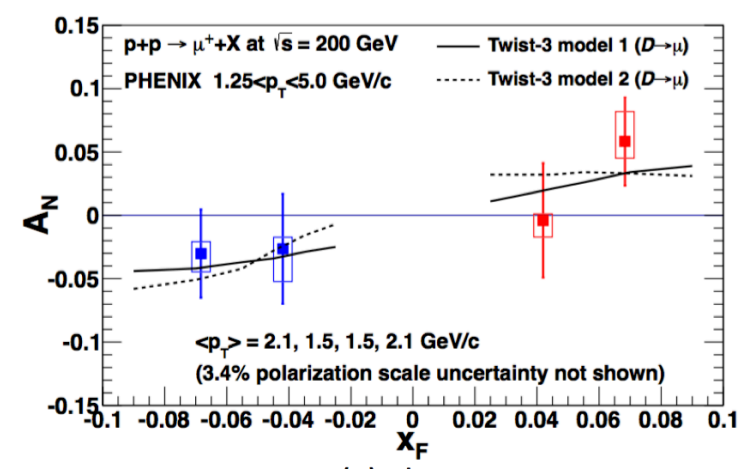

$(+)$ charge

Figure 2: Observed analyzing power $A_{\mathrm{N}}$ of $\mu^{-}$(left) and $\mu^{+}$(right) productions in $\mathrm{p}+\mathrm{p}$ collision at $\sqrt{s}=$ $200 \mathrm{GeV}$ in forward rapidity region plotted as a function of $x_{\mathrm{F}}$ [B]]. Theory curves are twist-3 three gluon correlation calculations [5].
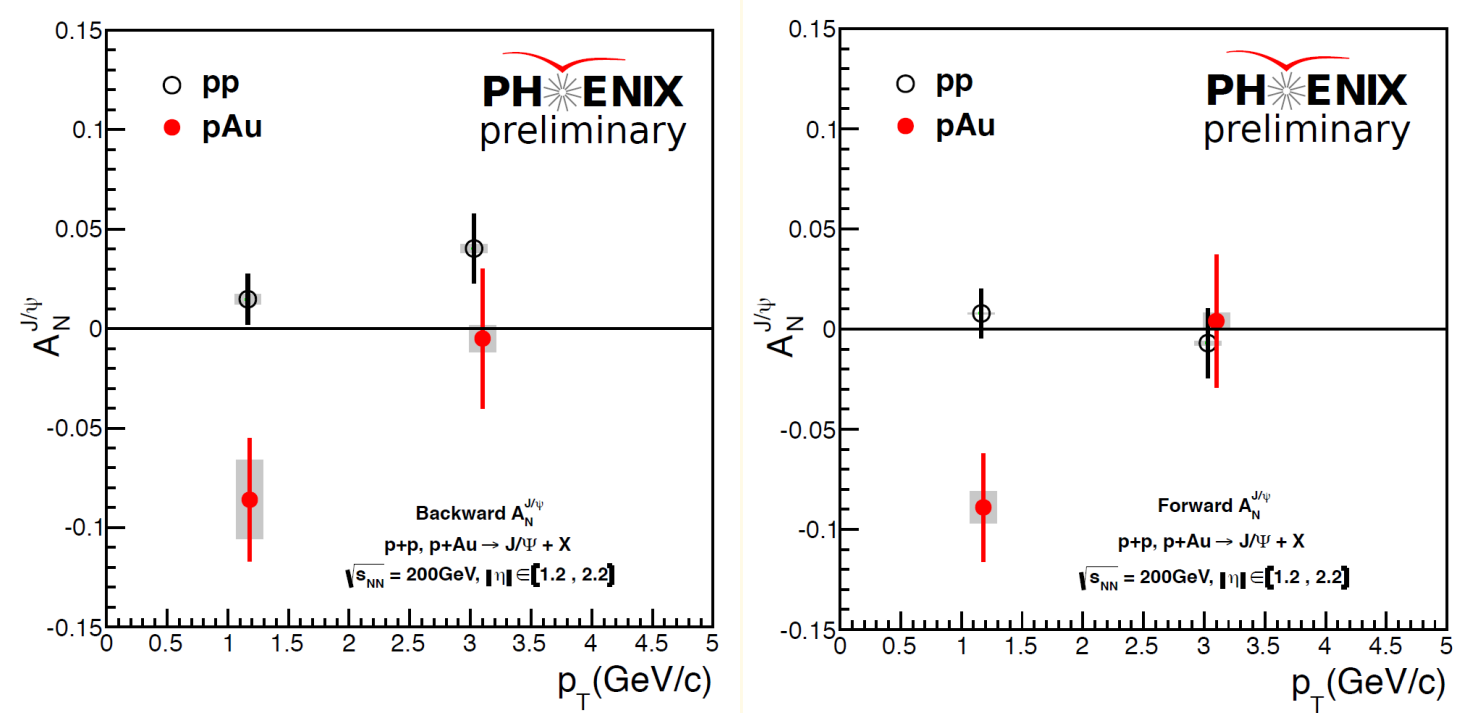

Figure 3: Observed analyzing power $A_{\mathrm{N}}$ of $J / \psi$ production in backward (left) and forward (right) productions in forward rapidity region plotted as a function of $p_{\mathrm{T}}$. Open (black) and solid (red) symbols represent $A_{\mathrm{N}}$ results of $\mathrm{p}+\mathrm{p}$ and $\mathrm{p}+\mathrm{Au}$ collisions at $\sqrt{s}=200 \mathrm{GeV}$, respectively.

collision in rapidity $(2.5<y<4)$ region plotted as a function average number of participants $N_{\text {part }}$ [ $[$ ]. Open black, solid blue, and solid red symbols represent results of $1 \leq p_{\mathrm{T}} \leq 8,0.3 \leq p_{\mathrm{T}}<1$, and $0 \leq p_{\mathrm{T}}<0.3 \mathrm{GeV} / \mathrm{c}$, respectively. The smallest $0 \leq p_{\mathrm{T}}<0.3 \mathrm{GeV} / \mathrm{c}$ data show as large as factor of 7(2) excess compared to larger $p_{\mathrm{T}}$ data points in 70\%-90\% (50\%-70\%) centrality class (the two lowest multiplicity classes). The authors claim the $J / \psi$ production cross section associated with observed excess is obtained under the hypothesis that coherent photoproduction of $J / \psi$ via ultra peripheral collision (UPC) is the underlying physics mechanism. The UPC effect is known to be observed in very forward rapidity region where corresponding impact parameter is larger than 
nuclear radius. The present rapidity region $(2.5<y<4)$ corresponds to about a half of the nuclear radius and therefore the UPC effect is unexpected to be observed.

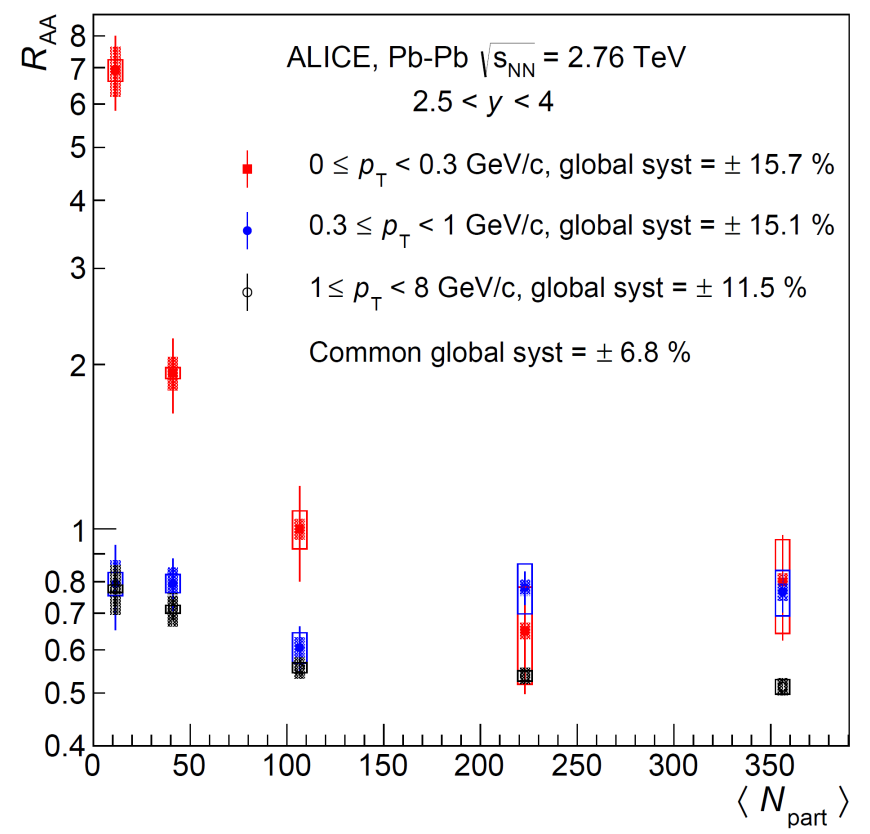

Figure 4: $R_{\mathrm{AA}}$ for $J / \psi$ production in $\mathrm{Pb}+\mathrm{Pb}$ collision at $\sqrt{2.76} \mathrm{TeV}$ observed in forward rapidity $(2.5<y<4)$ region plotted as a function average number of participants $N_{\text {part }}$ [目]. Open black, solid blue, and solid red symbols represent results of $1 \leq p_{\mathrm{T}} \leq 8,0.3 \leq p_{\mathrm{T}}<1$, and $0 \leq p_{\mathrm{T}}<0.3 \mathrm{GeV} / \mathrm{c}$, respectively.

\section{The Very Forward Rapidity}

Another example of larger asymmetry in $\mathrm{p}+\mathrm{A}$ than that of $\mathrm{p}+\mathrm{p}$ collision is the very forward neutron production [ $[$ ]. The rapidity is as forward as $6.8 \leq \eta \leq 8.8$ where the emission angle of neutron is less than $2.2 \mathrm{mrad}$. Relatively large energy neutrons are selected in the analysis, i.e. the neutron energy cut was applied at the energy fraction of incident proton $x_{\mathrm{F}}=E_{\mathrm{n}} / E_{\mathrm{p}} \geq 0.4$ [ [ $]$ ]. The production mechanism of such a neutron is driven by soft process and of which cross section is well described by one pion exchange (OPE) model. On the other hand, the asymmetry in $\mathrm{p}+\mathrm{p}$ was well explained by the interference between spin-flip $\pi$ exchange and spin non-flip $a_{1}$-Reggeon exchange which has a large phase shift in between[ [] . However, this hadronic interference model predicts only moderate $A$-dependence [ए]] and cannot explain the drastic A-dependence as shown in Fig. [. This unexpectedly drastic A-dependence is well explained by the UPC effect[[]] as demonstrated by open symbols. Due to our event selection of relatively large $x_{\mathrm{F}}$ neutron in the very forward rapidity region, the photon and momentum are biased to be as small as a couple hundred $\mathrm{MeV}$ where photo- $\Delta$ excitation of incoming polarized proton is known to play dominant role in the cross section. The forward neutron can be considered as either the decay product of $\Delta$ excitation or the consequence of the threshold non-resonant $\pi^{+}$production. The asymmetry of photo- $\pi^{+}$production from a transversely polarized proton target was measured at ELSA using PHOENICS-detector [ए2]. Significantly large asymmetry over $80 \%$ was reported below $\Delta$ region 
which indicates this is due to the interference between spin flipping $M_{1+}$ and non-spin flipping non-resonance $E_{0+}$ (Kroll-Rudermann term) amplitudes.

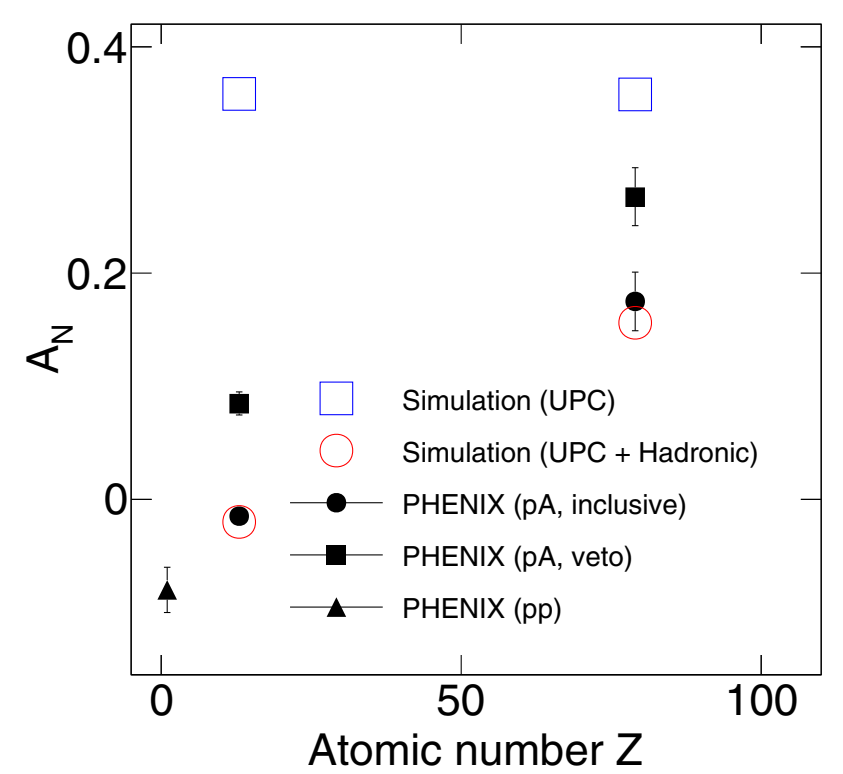

Figure 5: (Observed forward neutron $A_{\mathrm{N}}$ in transversely polarized proton (nucleus) collisions at at $\sqrt{s}=$ $200 \mathrm{GeV}$. Data points are $\mathrm{A}=1, \mathrm{~A}=27$, and $\mathrm{A}=197$ are results of $\mathrm{p}+\mathrm{p}$ (solid triangle), $\mathrm{p}+\mathrm{Al}$, and $\mathrm{p}+\mathrm{Au}$, respectively. Solid circle and Solid square data points are neutron inclusive, neutron + BBC veto events, respectively

In summary, the PHENIX collaboration explored A-dependence of transverse single spin asymmetries in wide rapidity region. Although no finite asymmetries are found in the central rapidity region $(-0.35<\eta<3.5)$, we observed unexpectedly strong A-dependence in forward $\mathrm{J} / \psi$ and the very forward neutron asymmetries. Important role of UPC effect is pointed out at least for very forward neutron asymmetries.

\section{References}

[1] B. E. Bonner et. al., Phys. Rev. Lett. 61, 1918 (1988).

[2] RHIC The Cold Nuclear Plan for 2017 to 2023, nucl-ex, arXiv:1602.03922 and the references therein.

[3] C. Aidala, et al. (PHENIX Collaboration), Phys. Rev. D 95, 112001 (2017).

[4] H. Akikawa et al., NIM A499, 537-548 (2003).

[5] Y. Koike and S. Yoshida, Phys. Rev. D 84, 014026 (2011).

[6] J. Adam, et al. (ALICE Collaboration), Phys. Rev. Lett. 116, 222301 (2016).

[7] B. Z. Kopeliovich, I. K. Potashnikova, and Ivan Schmidt: Phys. Rev. Lett. 64, 357 (1990).

[8] Y. Fukao et al., Phys. Lett. B 650, 325 (2007), A. Adare et al.: Phys. Rev. D 88, 032006 (2013).

[9] C. Aidala, et al. (PHENIX Collaboration), arXiv:1703.10941. 
[10] B. Z. Kopeliovich, et al. , arXiv:1702.07708.

[11] G. Mitsuka, Phys. Rev. C 95, 044908 (2017).

[12] H. Dutz, et al. , Nucl. Phys. A 601, (1996) 319-332. 